\title{
Onychomycosis: New Developments in Diagnosis, Treatment, and Antifungal Medication Safety
}

\author{
Shari R. Lipner, MD, PhD
}

$\square$ nychomycosis is the most prevalent nail condition worldwide and has a significant impact on quality of life. ${ }^{1}$ There were 10 million physician visits for nail fungal infections in the National Ambulatory Medical Care Survey from 2007 to 2016, which was more than double the number of all other nail diagnoses combined. ${ }^{2}$ Therefore, it is important for dermatologists to be familiar with the most current data on diagnosis and treatment of this extremely common nail disease as well as antifungal medication safety.

\section{Onychomycosis Diagnosis}

Diagnosis of onychomycosis using clinical examination alone has poor sensitivity and specificity and may lead to progression of disease and unwanted side effects from inappropriate therapy. ${ }^{3,4}$ Dermoscopy is a useful adjunct but diagnostically is still inferior compared to mycologic testing. ${ }^{5}$ Classical methods of diagnosis include potassium hydroxide staining with microscopy, fungal culture, and histopathology. Polymerase chain reaction is a newer technique with wide accessibility and excellent sensitivity and specificity. ${ }^{6}$ Although these techniques have excellent diagnostic accuracy both alone and in combination, the ideal test would have $100 \%$ sensitivity and specificity and would not require nail sampling. Artificial intelligence recently has been studied for the diagnosis of onychomycosis. In a prospective study of 90 patients with onychodystrophy who had photographs of the nails taken by nonphysicians, deep neural networks showed comparable sensitivity $(70.2 \%$ vs $73.0 \%)$ and specificity $(72.7 \%$ vs $49.7 \%)$ for diagnosis of onychomycosis vs clinical examination by dermatologists with a mean of 5.6 years of experience. ${ }^{7}$ Therefore, artificial intelligence may be considered as a supplement to clinical examination for dermatology residents and junior attending dermatologists and may be superior to clinical examination by nondermatologists, but mycologic confirmation is still necessary before initiating onychomycosis treatment.

\section{Treatment of Onychomycosis}

There are 3 topical therapies (ciclopirox lacquer $8 \%$, efinaconazole solution $10 \%$, and tavaborole solution $5 \%$ ) and 3 oral therapies (terbinafine, itraconazole, and griseofulvin) that are approved by the US Food and Drug Administration for onychomycosis therapy. Griseofulvin rarely is used due to the availability of more efficacious treatment options. Fluconazole is an off-label treatment that often is used in the United States. ${ }^{8}$

There are new data on the efficacy and safety of topical onychomycosis treatments in children. A phase 4 open-label study of efinaconazole solution $10 \%$ applied once daily for 48 weeks was performed in children aged 6 to 16 years with distal lateral subungual onychomycosis $(\mathrm{N}=62){ }^{9,10}$ The medication was both well tolerated and safe in children. The only treatment-related adverse event was onychocryptosis, which was reported by 2 patients. At week 52, mycologic cure was $65 \%$ and complete cure was $40 \%(\mathrm{~N}=50)$. In a pharmacokinetic assessment performed in a subset of 17 patients aged 12 to 16 years, efinaconazole was measured at very low levels in plasma. ${ }^{9}$

A phase 4 open-label study also was performed to evaluate the safety, pharmacokinetics, and efficacy of tavaborole for treatment of distal lateral subungual onychomycosis in children aged 6 years to under 17 years $(\mathrm{N}=55) .{ }^{11}$ Tavaborole solution 5\% was applied once daily for 48 weeks; at week 52, mycologic and complete cures were $36.2 \%$ and $8.5 \%$, respectively $(\mathrm{N}=47)$. Systemic exposure was low $\left(\mathrm{C}_{\max }=5.9 \mathrm{ng} / \mathrm{mL}\right.$ [day 29] $)$ in a subset of patients aged 12 years to under 17 years $(\mathrm{N}=37)$, and the medication demonstrated good safety and tolerability. ${ }^{11}$

Fosravuconazole was approved for treatment of onychomycosis in Japan in 2018. In a randomized, doubleblind, phase 3 trial of oral fosravuconazole $100 \mathrm{mg}$ once daily $(n=101)$ vs placebo $(n=52)$ for 12 weeks in patients with onychomycosis (mean age, 58.4 years), the complete cure rate at 48 weeks was $59.4 \% .^{12}$ In a small trial of

From the Department of Dermatology, Weill Cornell Medicine, New York, New York.

The author reports no conflict of interest.

Correspondence: Shari R. Lipner MD, PhD, 1305 York Ave, New York, NY 10021 (sh19032@med.cornell.edu).

doi:10.12788/cutis.0197 
37 elderly patients (mean age, 78.1 years), complete cure rates were $5.0 \%$ in patients with a nail plate thickness of $3 \mathrm{~mm}$ or greater and $58.8 \%$ in those with a thickness less than $3 \mathrm{~mm}$, and there were no severe adverse events. ${ }^{13}$ In addition to excellent efficacy and proven safety in elderly adults, the main advantage of fosravuconazole is lesspotent inhibition of cytochrome P450 3A compared to other triazole antifungals, with no contraindicated drugs listed.

\section{Safety of Antifungals}

There are new data describing the safety of oral terbinafine in pregnant women and immunosuppressed patients. In a nationwide cohort study conducted in Denmark $(1,650,649$ pregnancies [942 oral terbinafine exposed, 9420 unexposed matched cohorts]), there was no association between oral or topical terbinafine exposure during pregnancy and risk of preterm birth, small-for-gestationalage birth weight, low birth weight, or stillbirth. ${ }^{14}$ In a small study of 13 kidney transplant recipients taking oral tacrolimus, cyclosporine, or everolimus who were treated with oral terbinafine, there were no severe drug interactions and no clinical consequences in renal grafts. ${ }^{15}$

There also is new information on laboratory abnormalities in adults, children, and patients with comorbidities who are taking oral terbinafine. In a retrospective study of 944 adult patients without pre-existing hepatic or hematologic conditions who were prescribed 3 months of oral terbinafine for onychomycosis, abnormal monitoring liver function tests (LFTs) and complete blood cell counts (CBCs) were uncommon (2.4\% and $2.8 \%$, respectively) and mild and resolved after treatment completion. In addition, patients with laboratory abnormalities were an average of 14.8 years older and approximately 3 -times more likely to be 65 years or older compared to the overall study population. ${ }^{16}$ There were similar findings in a retrospective study of 134 children 18 years or younger who were prescribed oral terbinafine for superficial fungal infections. Abnormal monitoring LFTs and CBCs were uncommon $(1.7 \%$ and $4.4 \%$, respectively) and mild, resolving after after treatment completion. ${ }^{17}$ Finally, in a study of 255 patients with a preexisting liver or hematologic condition who were prescribed oral terbinafine for onychomycosis, worsening of LFT or $\mathrm{CBC}$ values were rare, and all resolved after treatment completion or medication discontinuation. ${ }^{18}$

\section{Final Thoughts}

Mycologic confirmation is still necessary before treatment despite encouraging data on use of artificial intelligence for diagnosis of onychomycosis. Efinaconazole solution 10\% and tavaborole solution 5\% have shown good safety, tolerability, and efficacy in children with onychomycosis. Recent data suggest the safety of oral terbinafine in pregnant women and kidney transplant recipients, but these findings must be corroborated before its use in these populations. Fosravuconazole is a promising systemic treatment for onychomycosis with no drug-drug interactions reported to date. While baseline laboratory testing is recommended before prescribing terbinafine, interval laboratory monitoring may not be necessary in healthy adults. ${ }^{19}$ Prospective studies are necessary to corroborate these findings before formal recommendations can be made for prescribing terbinafine in the special populations discussed above, including children, and for interval laboratory monitoring.

\section{REFERENCES}

1. Stewart CR, Algu L, Kamran R, et al. Effect of onychomycosis and treatment on patient-reported quality-of-life outcomes: a systematic review [published online June 2, 2020]. J Am Acad Dermatol. doi:10.1016/j. jaad.2020.05.143

2. Lipner SR, Hancock JE, Fleischer AB. The ambulatory care burden of nail conditions in the United States [published online October 21, 2019]. J Dermatolog Treat. doi:10.1080/09546634.2019.1679337

3. Lipner SR, Scher RK. Onychomycosis-a small step for quality of care. Curr Med Res Opin. 2016;32:865-867.

4. Lipner SR, Scher RK. Confirmatory testing for onychomycosis. JAMA Dermatol. 2016;152:847.

5. Piraccini BM, Balestri R, Starace M, et al. Nail digital dermoscopy (onychoscopy) in the diagnosis of onychomycosis. J Eur Acad Dermatol Venereol. 2013;27:509-513.

6. Lipner SR, Scher RK. Onychomycosis: clinical overview and diagnosis. J Am Acad Dermatol. 2019;80:835-851.

7. Kim YJ, Han SS, Yang HJ, et al. Prospective, comparative evaluation of a deep neural network and dermoscopy in the diagnosis of onychomycosis. PLoS One. 2020;15:e0234334.

8. Lipner SR, Scher RK. Onychomycosis: treatment and prevention of recurrence. J Am Acad Dermatol. 2019;80:853-867.

Eichenfield LF, Elewski B, Sugarman JL, et al. Efinaconazole 10\% topical solution for the treatment of onychomycosis in pediatric patients: openlabel phase 4 study [published online July 2, 2020]. J Am Acad Dermatol. doi:10.1016/j.jaad.2020.06.1004

10. Eichenfield LF, Elewski B, Sugarman JL, et al. Safety, pharmacokinetics, and efficacy of efinaconazole $10 \%$ topical solution for onychomycosis treatment in pediatric patients. J Drugs Dermatol. 2020;19:867-872.

11. Rich P, Spellman M, PurohitV, et al. Tavaborole $5 \%$ topical solution for the treatment of toenail onychomycosis in pediatric patients: results from a phase 4 open-label study. J Drugs Dermatol. 2019;18:190-195.

12. Watanabe S, Tsubouchi I, Okubo A. Efficacy and safety of fosravuconazole L-lysine ethanolate, a novel oral triazole antifungal agent, for the treatment of onychomycosis: a multicenter, double-blind, randomized phase III study. J Dermatol. 2018;45:1151-1159.

13. Noguchi H, Matsumoto T, Kimura U, et al. Fosravuconazole to treat severe onychomycosis in the elderly [published online October 25, 2020]. J Dermatol. doi:10.1111/1346-8138.15651

14. Andersson NW, Thomsen SF, Andersen JT. Exposure to terbinafine in pregnancy and risk of preterm birth, small for gestational age, low birth weight, and stillbirth: a nationwide cohort study [published online October 22, 2020]. J Am Acad Dermatol. doi:10.1016/j.jaad.2020.10.034

15. Moreno-Sabater A, Ouali N, Chasset F, et al. Severe onychomycosis management with oral terbinafine in a kidney transplantation setting: clinical follow-up by image analysis [published online November 27, 2020]. Mycoses. doi:10.1111/myc. 13220

16. Wang Y, Geizhals S, Lipner SR. Retrospective analysis of laboratory abnormalities in patients prescribed terbinafine for onychomycosis. J Am Acad Dermatol. 2021;84:497-499.

17. Wang Y, Lipner SR. Retrospective analysis of laboratory abnormalities in pediatric patients prescribed terbinafine for superficial fungal infections [published online January 27, 2021]. J Am Acad Dermatol. doi:10.1016/j. jaad.2021.01.073

18. Wang Y, Lipner SR. Retrospective analysis of laboratory abnormalities in patients with preexisting liver and hematologic diseases prescribed terbinafine for onychomycosis. J Am Acad Dermatol. 2021;84:220-221.

19. Lamisil. Prescribing information. Novartis Pharmaceuticals Corporation; 2010. https://www.accessdata.fda.gov/drugsatfda_docs /label/2010/022071s003lbl.pdf 